\title{
DIE AO AUSTRIA ALS QUALITÄTSSICHERUNGSAGENTUR FÜR DAS ÖSTERREICHISCHE HOCHSCHULSYSTEM
}

\author{
ACHIM HOPBACH \\ DOI: 10.22163/fteval.2019.448
}

$\mathrm{D}$ e A0 Austria wurde 2012 im Rahmen des Inkrafttretens des Hochschul-Qualitätssicherungsgesetzes (HS-OSG) als nationale Qualitätssicherungsagentur für das österreichische Hochschulsystem gegründet; in ihr wurden die drei bereits bestehenden Qualitätssicherungsagenturen, der Österreichischer Akkreditierungsrat für Privatuniversitäten (ÖAR), der Fachhochschulrat (FHR) und die nicht auf einen Sektor eingeschränkte österreichische Qualitätssicherungsagentur (AOA), fusioniert. Das HS-OSG definiert elf Aufgaben der AO Austria, die sich im Wesentlichen in vier Bereiche unterteilen lassen, nämlich die

- Entwicklung und Durchführung von Begutachtungsverfahren

- Erstellung von Analysen und Berichten

- Beratung und Information zu Fragen der Qualität und des Qualitätsmanagements an Hochschulen

- Aufsicht über akkreditierte Hochschulen und deren Studiengänge

zu denen als Querschnittsaufgabe die internationale Zusammenarbeit hinzutritt. ${ }^{1}$

Den größten Umfang der Tätigkeiten nehmen dabei die Begutachtungen von und an Hochschulen ein, nämlich die

- Begutachtung hochschulinterner Qualitätsmanagementsysteme im Rahmen des Audits an öffentlichen Universitäten und Fachhochschulen

- Begutachtung der gesamten Hochschulinstitution und ihrer Aktivitäten im Rahmen der institutionellen Akkreditierung von Privatuniversitäten und Fachhochschulen

- Begutachtung von Studiengängen im Rahmen der Studiengangakkreditierung an Privatuniversitäten und Fachhochschulen

- Begutachtung von Organisationseinheiten oder Studiengängen im Rahmen freiwilliger Evaluierungen und die

- Begutachtung von Lehrgängen der hochschulischen Weiterbildung im Rahmen einer freiwilligen Akkreditierung.

\section{GESETZLICHE}

RAHMENBESTIMMUNGEN
DER BEGUTACHTUNGEN

Das HS-OSG ist nicht nur Grundlage für die Einrichtung der A0 Austria, es regelt auch die Grundsätze der externen Qualitätssicherungsverfahren an öffentlichen Universitäten, Privatuniversitäten und Fachhochschulen. Unter anderem definiert es in § 2 Ziff. 2 zunächst allgemein, was Qualitätssicherungsverfahren sind, nämlich „formelle, durch unabhängige und externe Gutachterinnen und Gutachter durchgeführte Verfahren, die die Übereinstimmung von Bildungseinrichtungen und Studien oder des Qualitätsmanagementsystems der Bildungseinrichtungen mit definierten Kriterien und Standards feststellen." Unverkennbar dominiert die summative Funktion der externen Qualitätssicherung. Eine Spezifizierung erfolgt über die Konsequenzen der Begutachtungen: die Akkreditierung ist „die formelle staatliche Anerkennung einer Bildungseinrichtung (institutionelle Akkreditierung) oder von Studien (Programmakkreditierung) anhand von definierten Kriterien und Standards." Das Audit besitzt zwar auch eine Konsequenz, nämlich die Zertifizierung des Qualitätsmanagementsystems als „formelle Bescheinigung der Konformität des Qualitätsmanagementsystems einer Bildungseinrichtung mit definierten Kriterien und Standards" ${ }^{2}$; hiermit sind aber keine Rechtsfolgen verbunden.

Neben der Definition der Qualitätssicherung im Wesentlichen anhand des Zwecks, definiert das HS-OSG nur zwei Eckpunkte für die Durchführung der Verfahren, indem Gutachterinnen und Gutachter zu beteiligen und die Ergebnisse zu veröffentlichen sind. ${ }^{3}$

Desgleichen werden auch die Beurteilungskriterien und -standards weitgehend nicht per Gesetz definiert. Stattdessen bestimmt das HSOSG in §22 Abs. 2 Prüfbereiche für die Durchführung des Audits, in den $\S \S 23$ bzw. 24 Prüfbereiche für die Akkreditierung von Fachhochschulen bzw. Privatuniversitäten und jeweils deren Studiengänge. Allerdings enthalten die Gesetze für Privatuniversitäten (PUG) und Fachhochschulen 
(FHStG) weitere Bestimmungen, die für die Akkreditierung relevant sind und Anforderungen an die Hochschulen als Institution und an deren Studiengänge definieren.

Insgesamt beschränken sich die gesetzlichen Vorgaben somit auf grundlegende Rahmensetzungen, was der A0 Austria einen Gestaltungsspielraum für die Ausgestaltung der Begutachtungen gibt. Für die gesetzlich nicht verpflichtenden Begutachtungsverfahren macht das HSOSG keine Vorgaben.

\section{REFERENZPUNKTE FÜR DIE TÄTIGKEIT DER AO AUSTRIA}

Für die Tätigkeit der A0 Austria und somit auch die Ausgestaltung der Begutachtungen von und an Hochschulen sind vier Referenzpunkte besonders maßgeblich:

Ein zentraler Referenzpunkt für die Ausgestaltung der Begutachtungen von und an Hochschulen ist das mit dem HS-OSG verfolgte Ziel einer Harmonisierung der externen Qualitätssicherung im österreichischen Hochschulsystem. Durch die Überwindung der vormals bestehenden "starke(n) Zersplitterung der Gremien und Verfahren“ sollten "gemeinsame (Mindest-) Standards für hochschulische Angebote“, eine „Festlegung gemeinsamer Prüfbereiche für die Qualitätssicherungsverfahren, die sektorenübergreifend zur Anwendung kommen" und schließlich eine "sektorenübergreifende Einrichtung für die externe Qualitätssicherung" etabliert werden. ${ }^{4}$ In der sektorenübergreifenden Perspektive liegt somit ein wesentlicher Orientierungspunkt für die Agentur. Erstmals sollte die externe Qualitätssicherung für öffentliche Universitäten, Privatuniversitäten und Fachhochschulen harmonisiert werden, die Pädagogischen Hochschulen blieben jedoch unberücksichtigt.

Ein zweiter Referenzpunkt liegt in der Verbindung behördlicher und nicht-behördlicher Aufgaben in einer Qualitätssicherungsagentur. Bei der Akkreditierung von Privatuniversitäten und Fachhochschulen sowie deren Studiengängen wird die AO Austria als Behörde tätig, deren Entscheidungen gleichzeitig die staatliche Anerkennung der Institution und der von ihr vergebenen Hochschulgrade als österreichische Hochschulabschlüsse bedeutet. In Ergänzung übt die $\mathrm{AO}$ Austria auch eine Aufsicht über die akkreditierten Hochschulen insofern aus, als sie über die Einhaltung der Akkreditierungsbedingungen im Rahmen der Jahresberichte wacht. Im Unterschied hierzu sind die Auditverfahren zur Zertifizierung der internen Qualitätsmanagementsysteme an öffentlichen Universitäten und Fachhochschulen zwar auch verpflichtend, die A0 Austria wird hier aber nicht als Behörde, sondern als privater Dienstleister tätig. Hinzu kommt, dass die Auditverfahren wettbewerblich eingebettet sind, indem die Hochschulen auch andere Dienstleister wählen können, sofern sie den üblichen internationalen Standards genügen. Der Unterschied der Rechtsform der Begutachtungen wirkt sich auch auf deren Ausgestaltung aus, was im nächsten Kapitel näher betrachtet wird. In der gesetzlich determinierten Verbindung behördlicher und nicht-be- hördlicher Funktionen liegt ein Spezifikum, das die A0 Austria von den meisten anderen Qualitätssicherungsagenturen im Europäischen Hochschulraum unterscheidet.

Ein dritter Referenzpunkt ist die Ausdehnung des Aufgabenspektrums über die Durchführung von Begutachtungsverfahren hinaus. Durch die Aufgaben in den Bereichen Analyse und Berichtswesen wird der Fokus der Tätigkeit über eine einzelne Hochschule hinaus auf Teile des oder auf das gesamte Hochschulsystem gerichtet. In diesem Bereich befasst sich die $\mathrm{AO}$ Austria mit Fragestellungen, die sich auf mehrere Hochschulen, Teile einzelner Hochschulsektoren oder das gesamte Hochschulsystem beziehen (z. B. Qualitätssicherung von Berufungsverfahren, Qualitätssicherung von hochschulischer Weiterbildung usw.). Ein wesentliches Merkmal dieser Aktivitäten ist die Einbeziehung relevanter Akteure und Institutionen aus dem Hochschulsektor in die Entwicklung und Durchführung von Analysen. Eine weitere Gruppe von Analysen beruht auf den von der A0 Austria durchgeführten Qualitätssicherungsverfahren. Einerseits dienen die Ergebnisse aus diesen Analysen dazu, ein Verständnis für die aktuellen Problemfelder und Entwicklungen an den Hochschulen zu gewinnen. Andererseits werden die Ergebnisse und etwaige Empfehlungen veröffentlicht, um sie anderen Akteuren im Hochschulbereich oder anderen Interessengruppen zur Verfügung zu stellen.

Der vierte Referenzpunkt ist die auch durch das HS-OSG geforderte Einbettung der externen Qualitätssicherung in den Europäischen Hochschulraum, indem die Verfahren an internationaler guter Praxis ausgerichtet sind, wie sie insbesondere in den Standards and Guidelines for Quality Assurance in the European Higher Education Area (ESG) zum Ausdruck kommen. Zwecks Sicherstellung der korrekten Anwendung dieser Prinzipien und Standards muss die Agentur regelmäßig eine internationale Evaluierung durchlaufen. Im Jahr 2019 konnte die A0 Austria die letzte turnusgemäße Evaluierung erneut mit großem Erfolg abschließen. ${ }^{5}$

Diese Referenzpunkte finden sich auch im Leitbild der A0 Austria wieder, das die Gründungsphilosophie und das breite Aufgabenspektrum spiegelt:

„Die AQ Austria ist eine in Österreich und international anerkannte und in mehreren Ländern tätige Qualitätssicherungsagentur. Sie richtet sich nach den Werten Öffentliche Verantwortung für die Qualität in der Hochschulbildung, Sicherung der akademischen Freiheit, Autonomie der Hochschulen und Wissenschaftliche Integrität. Sie unterstützt die Hochschulen in der kontinuierlichen Qualitätsentwicklung und gibt aufgrund ihrer Expertise Impulse für die Weiterentwicklung des Hochschulsystems. Zudem leistet sie einen Beitrag zur transparenten Information über die Qualität der Hochschulen in ihren Leistungsbereichen. (...)"

Für die AO Austria ist ihr breites Aufgabenspektrum, vor allem die Tätigkeit im Bereich Analysen und Berichte eine zentrale Komponente ihrer Rolle als nationale Qualitätssicherungsagentur, die nicht von ihrer Aufgabe der Begutachtung von Hochschulen und Studiengängen getrennt werden kann. Nach Auffassung der A0 Austria sollte eine Qualitätssicherungsagentur immer mehr sein als lediglich Organisatorin von Begutachtungen. 


\section{AUSGESTALTUNG DER BEGUTACHTUNGSVERFAHREN DURCH DIE AO AUSTRIA}

Grundsätzlich haben sämtliche gesetzlich verpflichtende Begutachtungsverfahren drei Gemeinsamkeiten:

- Die Beurteilung, ob der Gegenstand - Programm, Institution oder internes Qualitätsmanagementsystem - vordefinierte Standards oder Kriterien erfüllt. Eine Ausnahme bilden hier die freiwilligen Evaluierungen von Studiengängen und organisatorischen Einheiten, die in der Regel Potenziale zur künftigen Weiterentwicklung im Fokus haben.

- Die Unterstützung der Hochschulen bei der Qualitätsentwicklung.

- Die Übereinstimmung der Verfahrensschritte mit internationaler guter Praxis: Selbstevaluierung, externe Begutachtung durch Gutachterinnen und Gutachter, Vor-Ort-Besuch, veröffentlichtes Gutachten und Follow-up-Maßnahmen.

Auf dieser gemeinsamen Grundlage variiert die A0 Austria die Begutachtungen je nach deren Zweck und Gegenstand, wobei die Varianten im Wesentlichen die Standards oder Beurteilungskriterien betreffen; die Verfahrensschritte weisen nur marginale Unterschiede auf.

Für die Ausgestaltung der Begutachtungsverfahren ist der grundlegende Unterschied zwischen Audit- und Akkreditierungsverfahren hinsichtlich der Begutachtungsgegenstände und der Rechtsfolgen von zentraler Bedeutung:

Während es in der Akkreditierung um die unmittelbare Beurteilung der Qualität der Hochschulen in Forschung und Lehre sowie den weiteren Leistungsbereichen geht, fokussiert das Audit nur mittelbar auf die Qualität der Hochschulen, indem die internen Strukturen und Prozesse zur Erzeugung und Gewährleistung der Qualität beurteilt werden. Dieser grundlegende Unterschied schlägt sich unmittelbar in der Ausgestaltung der jeweiligen Beurteilungskriterien oder Standards nieder.

Für die Beurteilung, ob die internen Strukturen und Prozesse des Qualitätsmanagements geeignet sind, die angestrebte und erforderliche Qualität zu erzeugen und zu gewährleisten, verwendet die A0 Austria im Rahmen des Audits fünf eher allgemein formulierte Standards, die zentrale Aspekte eines Qualitätsmanagementsystems, wie die Festlegung von Verantwortlichkeiten und Strukturen, dessen nachweisbare Wirksamkeit und dessen Weiterentwicklung adressieren. ${ }^{7}$ Dass die Standards wenig detailliert sind und auf einer allgemeinen Ebene der Prinzipien verbleiben, ist notwendig und dem Begutachtungsgegenstand geschuldet. Da sich Hochschulen hinsichtlich Profil, Größe, Struktur und interner Kultur erheblich voneinander unterscheiden und die internen Qualitätsmanagementsysteme auf die jeweiligen Spezifika hin ausgerichtet sein müssen, kann es sinnvollerweise keine über Prinzipien hinausgehenden Detailvorgaben geben. Der Begutachtungsgegenstand und die, zumindest für Universitäten fehlende Rechtsfolge des Audits prädestinieren das Begutachtungsverfahren geradezu dafür, die Entwicklungsdimensi- on zu betonen. Dies tut die A0 Austria, indem sie auf die Wirksamkeit der Prozesse abzielt.

Es kann kaum verwundern, dass die Beurteilungskriterien in der Akkreditierung umfassender und detaillierter sind, da sie die Qualität der Leistungen der Hochschule mit all ihren Tätigkeitsbereichen oder eines Studiengangs mittelbar adressieren und außerdem die verschiedenen formalen Aspekte bzw. gesetzlichen Anforderungen, die auch für die Zulassung der Hochschulen und ihrer Studiengänge relevant sind, umfassen. Außerdem handelt es sich um staatliche Anerkennungsverfahren wodurch die Hochschulen an größtmöglicher Konkretisierung interessiert sind, auch wenn dies vordergründig zur Einengung von Gestaltungsspielräumen auf Seiten der Hochschulen führt. Darüber hinaus enthalten die Kriterien Elemente, die der Dimension der Qualitätsentwicklung dienen, wie z.B. die Ausgestaltung und Anwendung interner Qualitätssicherungsprozesse zur Weiterentwicklung von Studiengängen usw. Im höheren Detaillierungsgrad liegt ein typischer Unterschied zum Audit. Gemeinsam ist beiden Begutachtungen allerdings, dass die Kriterien bzw. Standards so ausformuliert sind, dass sie eine Ja/Nein-Entscheidung ermöglichen, da das Audit zwar keine Rechtsfolgen besitzt, aber zu einer Zertifizierungsentscheidung führt.

In den freiwilligen Begutachtungsverfahren stellt sich die Situation zum Teil anders dar. Die Verfahren zur freiwilligen Akkreditierung von Lehrgängen der hochschulischen Weiterbildung weisen einerseits sehr große Ähnlichkeiten mit den verpflichtenden Verfahren der Studiengangakkreditierung auf und berücksichtigen lediglich den Charakter der Weiterbildung, allerdings in ihrer hochschulischen Ausprägung. Auch wenn die Kriterien sehr ähnlich sind, liegt andererseits ein wesentlicher Unterschied darin, dass die Verfahren erst durchgeführt werden, wenn eine Kohorte den Lehrgang bereits absolviert hat. Diese Einschränkung ist der Betonung der Qualitätsentwicklung geschuldet. Da mit der freiwilligen Akkreditierung keine staatliche Anerkennung oder Einrichtungsgenehmigung verbunden ist, wählte die A0 Austria im Unterschied zu den exante Begutachtungen in den verpflichtenden Akkreditierungsverfahren die ex-post - Begutachtung, um auf der Grundlage der tatsächlichen Erfahrungen in der Durchführung des Lehrgangs die Qualitätsentwicklung in das Zentrum der Begutachtung zu stellen.

Gänzlich anders werden in der Regel die Standards oder Beurteilungskriterien in den freiwilligen Evaluierungen von Organisationseinheiten oder Studiengängen ausgestaltet. Da es hierbei zumeist ausschließlich um Meinungen von Expertinnen und Experten zu Entwicklungsstand und Entwicklungsperspektiven geht, werden die Referenzpunkte für jedes Verfahren individuell und gemeinsam mit der auftraggebenden Institution festgelegt. Lediglich die Verfahrensschritte sind insofern vorbestimmt, als sie den üblichen internationalen Standards genügen müssen.

Im Folgenden sollen drei Aspekte der Ausgestaltung der Begutachtungsverfahren erörtert werden, die auch in der Überarbeitung der Begutachtungsverfahren zwischen 2016 und 2018 eine zentrale Rolle spielten:

\section{SEKTORENÜBERGREIFENDE AUSGESTALTUNG}

Entsprechend dem Ziel des HS-OSG, die starke Zersplitterung der Verfahren zu überwinden, gestaltet die A0 Austria die Begutachtungsver- 
fahren so einheitlich wie möglich. Allerdings sind drei Einschränkungen zu machen:

Erstens ist festzustellen, dass das HS-OSG zwar nur wenige Vorgaben für die Ausgestaltung der Begutachtungsverfahren macht, die sektorspezifisch unterschiedlichen Zweckbestimmungen jedoch der Überwindung der Zersplitterung Grenzen setzt. Vor allem die Koexistenz von Akkreditierungs- und von Auditverfahren, somit Verfahren mit Zulassungsfunktion und Verfahren ohne jegliche Rechtsfolge (zumindest für Universitäten) zeigt, dass das HS-OSG den Verfahrenstypus nach wie vor im Wesentlichen entlang der Grenzen der drei Hochschulsektoren und nach den Rechtsfolgen bestimmt, was zu einer Teil-Vereinheitlichung der Verfahren für nur jeweils zwei Sektoren führt. Es kann nicht verwundern, dass es sich dabei zum einen hinsichtlich der Akkreditierung um die beiden privaten Sektoren der Privatuniversitäten und Fachhochschulen handelt, da die Verfahren auch die staatliche Anerkennung der Institution und ihrer Studienangebote umfasst. Zum anderen sind dies hinsichtlich des Audits die öffentlichen Universitäten und die Fachhochschulen, was insofern interessant ist, da sich die Rechtsfolgen erheblich voneinander unterscheiden. Für öffentliche Universitäten gibt es keine, für die Fachhochschulen ist die staatliche Anerkennung mit einem erfolgreichen Audit verknüpft. $^{8}$

Ihre Aufgabe, die Qualitätssicherungsverfahren zu entwickeln, nutzte die A0 Austria in Anlehnung an dieses Ziel des HS-OSG, indem sie die Kriterien für die Akkreditierung von Privatuniversitäten und Fachhochschulen und deren Studiengängen so weit als möglich und so weit als sinnvoll angesichts der unterschiedlichen Hochschultypen und Bildungsaufträge definiert hat. So basieren z.B. die Akkreditierungskriterien für Studiengänge unabhängig davon, ob diese von Privatuniversitäten oder von Fachhochschulen angeboten werden, immer auf demselben Fragenkanon:

- Sind für den geplanten Studiengang dem Qualifikationsprofil entsprechende Qualifikationsziele definiert?

- Wird das Erreichen der Qualifikationsziele durch ein geeignetes Studiengangkonzept (Curriculum) unterstützt?

- Sind Lehre, Lernprozess und Prüfung geeignet, um das Erreichen der Qualifikationsziele zu unterstützen?

- Stehen die erforderlichen Ressourcen (personelle, räumliche und sachliche) zur Verfügung?

- Wird in geeigneter Weise über Inhalte und Anforderungen des Studiengangs informiert?

- Werden geeignete Qualitätssicherungsmaßnahmen durchgeführt?

Die Unterschiede sind vergleichsweise gering und beziehen sich in der Regel auf nicht an beiden Hochschultypen vorhandene Begutachtungsbereiche, wie z.B. Doktoratsstudiengänge, die es an Fachhochschulen nicht gibt, oder auf spezifische Aspekte, die aus dem gesetzlich definierten Bildungsauftrag resultieren, so z.B. die Angewandte Forschungs- und Entwicklungsarbeit an Fachhochschulen, die nicht als gesetzliche Vorgabe für Privatuniversitäten existiert. Dort, wo es sich um die gleichen Begutachtungsgegenstände handelt, wurden kaum Unterschiede gemacht.
Im Falle der Audits ist die Übereinstimmung der Standards noch gröBer, was angesichts des Begutachtungsgegenstandes kaum verwundern kann. Da im Fokus des Audits hochschulinterne Steuerungsprozesse und diesbezüglich Verantwortlichkeiten und Zuständigkeiten stehen, ist eine sektorenspezifische Ausgestaltung nicht erforderlich. Nicht einmal die sektorenspezifischen Bestimmungen im HS-OSG, wonach an Fachhochschulen auch die Weiterbildungslehrgänge und an öffentlichen Universitäten auch Lehramtsstudiengänge von den jeweiligen Qualitätsmanagementsystemen und somit vom Audit erfasst sein müssen, machte eine Differenzierung erforderlich.

Einschränkend ist entsprechend der unterschiedlichen Gegenstände und Zweckbestimmungen der externen Qualitätssicherung zu bemerken, dass das Ziel des HS-OSG, „gemeinsame (Mindest-) Standards für hochschulische Angebote" zu etablieren, durch die Begutachtungsverfahren alleine notwendigerweise nur teilweise erreicht werden kann, da sich die Kriterien der Akkreditierung direkt auf die Qualität der Leistungen beziehen, die Standards der Auditverfahren auf Prozesse zur Sicherung der Qualität und nicht auf die Qualität hochschulischer Angebote an sich beziehen. In diesem Zusammenhang sollte die Bedeutung eines sektorübergreifenden Dialogs über Qualitätsstandards für hochschulische Angebote nicht unterschätzt werden.

\section{ENTWICKLUNGSDIMENSION VERSUS KONTROLL- DIMENSION}

Obwohl sich die Zweckbestimmungen und vor allem die Rechtsfolgen der Begutachtungsverfahren unterscheiden, und diese sich typischerweise an unterschiedlichen Stellen des Kontinuums zwischen den Polen Entwicklungsdimension und Kontrolldimension befinden, ist die A0 Austria bestrebt, der Qualitätsentwicklung einen möglichst hohen Stellenwert einzuräumen. Wie stark die Entwicklungsdimension unterstützt wird, hängt dabei auch vom Begutachtungsgegenstand ab. Bei Audits und institutionellen Akkreditierungen ist dies unkompliziert, da die internen Verfahren und Instrumente zur Qualitätsentwicklung einen Schwerpunkt der Begutachtung bilden. Insbesondere die Neugestaltung der institutionellen Reakkreditierung 2019 folgt diesem Weg, indem die Wirksamkeit des internen Qualitätsmanagements stärker in den Mittelpunkt gerückt wird. Bei Verfahren auf Programmebene wird die Entwicklungsdimension direkter adressiert, indem Empfehlungen für die Weiterentwicklung der Programme gegeben werden. Grundsätzlich wird diese Richtung auch durch die Neugestaltung der Gutachten bei institutionellen Reakkreditierungen unterstrichen, indem die seit der letzten Überprüfung erzielten Fortschritte hervorgehoben und Beispiele guter Praxis und Empfehlungen zur Weiterentwicklung aufgeführt werden.

\section{FOLLOW-UP}

Von zentraler Bedeutung für die Ausgestaltung externer Qualitätssicherungsverfahren ist der Standard, Begutachtungsverfahren nicht als punktuelles Ereignis durchzuführen, sondern in einen kontinuierlichen Entwicklungsprozess einzubetten. Ein zentrales Instrument hierfür ist

8 Siehe hierzu auch Achim Hopbach (2014), Externe Qualitätssicherung in Österreich, in: Winnfried Benz et al. (Hg.): Handbuch Qualität in Studium und Lehre. Berlin: Raabe, F1-A2. B 5, 107-128. 
das regelmäßige Follow-up eines Begutachtungsverfahrens, in dem verfolgt wird, wie die Ergebnisse des Verfahrens umgesetzt werden. In der Ausgestaltung des Follow-up sind der AO Austria durch das HS-OSG enge Grenzen gesetzt. Die Grundlage für das Follow-up wird in den Begutachtungsverfahren selbst bereitet, indem die Gutachterinnen und Gutachter angehalten sind, in den Gutachten Empfehlungen für weitere Entwicklungen zu geben. In Reakkreditierungen und bei Audits kommt hinzu, dass die A0 Austria Akkreditierungen unter Auflagen aussprechen kann, die in einem vorgegebenen Zeitraum zu erfüllen sind. Das HS-QSG sieht dieses Instrument allerdings nicht für erstmalige Akkreditierungen vor, worin eine Einschränkung zu sehen ist, die die A0 Austria in der Stärkung der Entwicklungsdimension behindert.

Zwar ist das Instrument der Auflagenerteilung das am weitesten verbreitete Follow-up Instrument in Akkreditierungsverfahren, es ist aber nicht das einzige. Bei den Privatuniversitäten und Fachhochschulen geht das gesetzliche Mandat der A0 Austria über Akkreditierungsentscheidungen hinaus und sieht ein weiteres Instrument vor, das eine wichtige Rolle für das Follow-up spielt. So müssen die Hochschulen jährlich einen Bericht über die wichtigsten internen Entwicklungen erstellen, wie z.B. die Umsetzung der Ergebnisse aus Begutachtungsverfahren.

Auch bei Audits setzt die AO Austria die Auflagenerteilung als ein Instrument des Follow-Ups ein ${ }^{9}$, zusätzlich werden aber den HochschuIen freiwillige und maßgeschneiderte Follow-up-Workshops angeboten. Der Zweck dieser Workshops besteht darin, über die Erörterung etwaiger Auflagen hinauszugehen und die im Gutachten enthaltenen Empfehlungen und mögliche Wege zu ihrer Umsetzung zu erörtern. Obwohl die A0 Austria von der Relevanz solcher Workshops überzeugt ist, hat sie bewusst darauf verzichtet, diese als regelhaften Schritt in den AuditProzess einzubeziehen. Der Grund ist die Tatsache, dass die A0 Austria bei Audits in Österreich mit ausländischen Qualitätssicherungsagenturen konkurrieren muss, von denen keine eine solche Maßnahme als verbindlichen Verfahrensschritt definiert hat. Da Arbeitsaufwand, Dauer und Kosten eines Prozesses relevante Kriterien für die Auswahl einer Agentur sind, wäre es für $\mathrm{A} 0$ Austria nicht vorteilhaft, einen obligatorischen Nachbereitungsworkshop zu integrieren.

\section{RESÜMEE}

Die A0 Austria ist zum einen eine „klassische“ Qualitätssicherungsagentur mit einer gesetzlich definierten Zuständigkeit für die verbindliche externe Qualitätssicherung im österreichischen Hochschulsystem, wobei die Pädagogischen Hochschulen unberücksichtigt bleiben. Ihre Besonderheiten resultieren aus dem „Gründungsauftrag" der Agentur vor dem Hintergrund der rechtlichen Ausgestaltung der verbindlichen externen Qualitätssicherung. Auch wenn „gemeinsame (Mindest-) Standards für hochschulische Angebote" und die Aufhebung der "starke(n) Zersplitterung der Gremien und Verfahren" Kernanliegen bei der Einrichtung der Agentur waren und die A0 Austria sich in der konzeptionellen Ausgestaltung und Durchführung ihrer Aktivitäten diesen Anliegen verpflichtet fühlt, setzen die gesetzlichen Bestimmungen zu Zweck, Begutachtungsgegenstand und Rechtsfolgen der verbindlichen externen Qualitätssicherung diesem Anliegen enge Grenzen, da diese sich nach wie vor von Hochschulsektor zu Hochschulsektor unterscheiden. Dies zeigt, dass durch die Begutachtungsverfahren alleine das Ziel des HS-OSG nicht erreicht werden kann, sondern die Tätigkeiten der A0 Austria im Bereich der hochschul- und sektorenübergreifenden Analysen und der Förderung der sektorenübergreifenden Kommunikation einen wesentlichen und notwendigen Beitrag hierzu leisten müssen.

\section{QUELLEN}

Achim Hopbach (2014), Externe Qualitätssicherung in Österreich, in: Winnfried Benz et al. (Hg.): Handbuch Qualität in Studium und Lehre. Berlin: Raabe, F1-A2. B 5, 107-128

Eva Erlinger-Schacherbauer (2013), Positionen des Bundesministeriums für Wissenschaft und Forschung (BMWF) zur Neuordnung der externen Qualitätssicherung in Österreich, in: Winnfried Benz et al. (Hg.): Handbuch Qualität in Studium und Lehre. Berlin: Raabe, B 5.1.

\section{AUTOR}

\section{DR. ACHIM HOPBACH}

Geschäftsführer AQ Austria

Franz-Kleingasse 5, 1190 Wien

E: achim.hopbach@aq.ac.at

9 Gemäß Richtlinie für das Audit des hochschulinternen Qualitätsmanagements der A0 Austria müssen Auflagen innerhalb von 2 Jahren als erfüllt nachgewiesen werden. https://www.aq.ac.at/de/audit/dokumente-audit-verfahren/Auditrichtlinie_2018.pdf?m=15508505178, abgerufen am 19.11.2019 